\title{
Revalorización de la cadena agroalimentaria a través de la innovación: Grupo La Caña
}

\author{
Beatriz Molina ${ }^{2}$ \\ Autor de Correspondencia: beatriz.molina@grupolacana.com
}

\section{Resumen:}

Actualmente los conceptos de bioeconomía, economía circular y sostenibilidad se han convertido en tendencia. Nos podríamos preguntar si realmente hay empresas que se basan en estos conceptos para marcar su estrategia y si se da la suficiente importancia a la sostenibilidad a nivel estratégico dentro de una empresa (Junta de Andalucía, 2018). La respuesta es que sí, tanto a nivel normativo como a nivel legislativo, la orientación de las empresas va en esta dirección. Como ejemplo de ello, se expone el caso del Grupo La Caña, empresa productora, comercializadora, exportadora e importadora de productos hortofrutícolas que tiene su sede en Costa Tropical de Granada, y trabaja en toda la región de Andalucía. A través del desarrollo socioeconómico sostenible, destaca su modelo de negocio mediante el uso eficiente de herramientas y recursos naturales. Por ello, ha sido crucial llevar a cabo una optimización de procesos, métodos y protocolos que proporcionen bienes y servicios adecuados para la gestión de la industria, así como reducir los residuos generados y su efecto en el entorno más inmediato.

Palabras clave: Bioeconomía, Economía circular, Sostenibilidad, Industria agroalimentaria, Alimentación funcional

\section{Enhancing the agri-food chain through innovation: Grupo La Caña}

\section{Beatriz Molina ${ }^{1}$}

\section{Abstract:}

The concepts of the bioeconomy, the circular economy and sustainability are timely ones today, prompting us to ask whether there really are companies that are based on them, with these ideas shaping their strategies and them assigning enough importance to sustainability at a strategic level (Junta de Andalucía, 2018). The answer is yes, at both the normative and legislative levels, as the orientations of companies are, in fact, headed in this direction. As an example of this, the Grupo La Caña is presented, a producer, marketer, exporter and importer of horticultural products based on Granada's "Tropical Coast" and operating throughout the Andalusia region. Through sustainable socioeconomic development, it stands out for a business model characterised by its efficient use of tools and natural resources. Hence, it has been crucial to carry out an optimisation of the processes, methods and protocols that provide goods and services well-adapted to the management of the industry, as well as to reduce waste generated and its effect on the immediate environment.

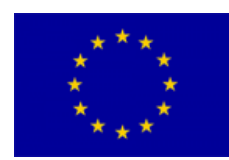
UNIOON EUROPEA

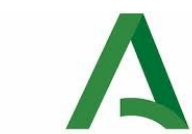

Junta de Andalucía
Andalucía

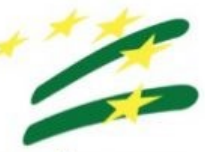

se mueve con Europa 
Keywords: Bioeconomy, Circular economy, Sustainability, Agri-food industry, Functional food

' Grupo Empresarial La Caña, Motril, Granada (ESPAÑA), beatriz.molina@grupolacana.com

\section{EJEMPLOS DE APLICACIÓN DE LA BIOECONOMÍA: PROYECTOS DE I+D+i DE GRUPO LA CAÑA}

Actualmente las líneas estratégicas de Investigación, Desarrollo e innovación se traducen en proyectos encaminados a una producción de alimentos más limpia (reduciendo la cantidad de subproductos o coproductos), sostenible, eficiente y con el fin último de volver al inicio del ciclo del producto. Las líneas de trabajo marcadas para llevar a cabo estas premisas se basan en el aprovechamiento máximo de materias primas, revalorización de subproductos o coproductos procedentes de la actividad, cuidado y conservación del medio ambiente. A continuación, se mostrarán algunos de los proyectos de l+D+i de Grupo La Caña a modo de ejemplo de aplicación de la bioeconomía.

\subsection{Proyecto BioREFINA}

El proyecto BioREFINA, es una iniciativa que surge al tomar consciencia del volumen de producto de categoría no comercial que se genera en Grupo La Caña actualmente. En este sentido, la industria agroalimentaria aborda como principal objetivo de este proyecto el aprovechamiento de restos vegetales tales como podas, restos de frutos y restos orgánicos (Kennet \& Winterhalder, 2006) procedentes de la actividad de la empresa desde el campo hasta la propia industria. Éstos, unidos a los residuos orgánicos procedentes de la ganadería, como los purines de cerdo, tienen como fin último ser transformados para su uso como biofertilizante, compost e incluso biogás.

La creación de biorrefinerías es uno de los resultados potenciales del proyecto a modo de instalaciones para transformar biomasa en bioenergía y/o bioproductos sostenibles. Actualmente se sigue trabajando en esta línea en la que además de resolver las limitaciones surgidas en la vida del proyecto, se plantea un escenario en el que se integra a productores de distintos ámbitos del sector hortofrutícola a nivel nacional. 
Figura 1. Biodigestor

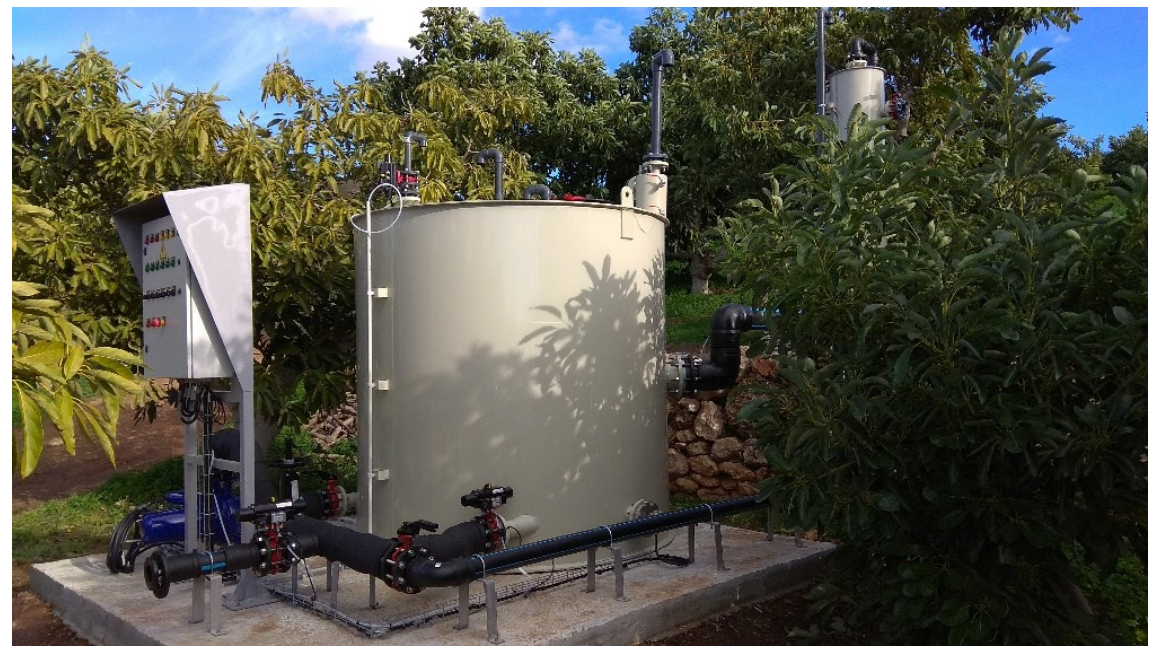

\subsection{Proyecto "Utilización eficiente del agua en cultivos hortícolas bajo invernadero"}

En relación con el consumo de agua, la actividad agrícola realiza un consumo estimado del $70-75 \%$ del total, quedando el resto para red urbana e industria. En concreto, Andalucía Oriental, cuenta con una superficie de cultivo intensivo bajo invernadero de más de 35.000 hectáreas que están repartidas entre las provincias de Almería, Granada y Málaga. En cada campaña de cultivo se producen alrededor de 6 millones de kilos de hortalizas. Como consecuencia de ello, cualquier mejora en la eficiencia provoca un impacto directo en el uso de agua y su disponibilidad. En este sentido, el Grupo Operativo Regional: "Utilización eficiente del agua en cultivos hortícolas bajo invernadero" ha permitido establecer estrategias de riego que minimicen el gasto de agua en cultivos hortícolas de invernadero, utilizando para ello sensores de bajo coste.

La utilización de sistemas que permitan controlar el uso de agua, el control de nutrientes, así como los lixiviados que se generan, la producción y factores ambientales del cultivo, permite que se optimice su uso de manera eficiente, tanto a nivel medioambiental como a nivel económico. El proyecto promueve una agricultura de precisión con un máximo aprovechamiento de agua de riego y uso eficiente de insumos (fertilizantes y bioestimulantes), que persigue reducir la contaminación de los recursos naturales, y contribuir al 
mantenimiento de los ecosistemas naturales y la sostenibilidad ambiental (Harris, 1989).

\subsection{Proyecto AVOCEMTUM}

Dentro de las líneas de revalorización de subproductos, destaca el proyecto AVOCEMTUM, materializado en una determinada gama de productos en el mercado, concretamente en el uso de aguacates para el desarrollo de guacamoles funcionales con propiedades saludables mediante la incorporación de nuevos ingredientes obtenidos a partir de la revalorización de coproductos. Esta gama destaca por ser funcional, para lo que Grupo La Caña se ha centrado en la obtención de un producto con propiedades saludables para el consumidor como sería el contenido del mismo en antioxidantes, su contenido en fibra o la disminución de su contenido de grasas.

La alimentación está dando un giro de 360 grados, pasando de productos sofisticados o ready to eat a lo que hoy comúnmente consideramos como consumo de productos tradicionales y productos frescos de origen agrícola y ganadero, base primaria de nuestra alimentación. Pero żpodría considerarse que estos recursos primarios son ilimitados? ¿̇s posible obtener todo lo que queremos y cuando queremos de los recursos naturales? A estas preguntas se puede responder de la siguiente manera: sabemos que los recursos son limitados, a lo que debemos sumar el crecimiento de población mundial; por ello tanto la sociedad, las administraciones como el sector, tienen la obligación de (i) tomar consciencia de las limitaciones de los recursos naturales y del impacto de su explotación (ii) planificar estratégicamente las políticas y los procedimientos de las industrias y (iii) distribuir la alimentación de la manera más eficiente posible, reduciendo el desperdicio alimentario y garantizando el hambre cero. Por eso desde Grupo La Caña, conscientes de que hay que buscar alternativas al consumo de alimentos tal y como se concibe actualmente, y continuando con la fuerte apuesta por la mejora de las cualidades organolépticas de sus productos frescos, se da un paso más dirigido a alimentos procesados que, encontrándose mínimamente procesados en una 
determinada etapa, mantienen sus propiedades organolépticas y puede alargarse su vida útil. Son los productos denominados de quinta gama.

El incremento de la población y el cambio climático mundial son obstáculos significativos que implican un compromiso de producción agrícola sostenible en el tiempo, debido a las limitaciones de la tierra cultivable disponible. De este modo, se hace necesario explorar nuevas fuentes de alimentación que tengan una alta capacidad de producción, no requiera fertilización, y sea menos sensible a efectos medioambientales, como son la sequía o a las plagas y enfermedades. Unido a esto, se detecta una reducción del consumo de carne en las dietas occidentales, asociada a connotaciones de salud y tendencias de vegetarianismo y veganismo, detectándose por tanto una oportunidad de mercado dada la creciente demanda de productos vegetales como alternativa.

Como se aprecia en la figura 2, en el marco de este proyecto se han determinado distintos formatos de Guacamoles (original premium, light, fibra y antioxidante), todo ello en colaboración con la Universidad de Granada y el Centro Tecnológico del Alimento Funcional (CIDAF). Con estas premisas se ha trabajado a escala laboratorio en la formulación de las matrices alimentarias en donde se han obtenido cinco recetas para cada una de ellas. Cada receta se ha sometido a distintos análisis para determinar su calidad microbiológica, nutricional-composicional y caracterización fisicoquímica. Sobre estos formatos a su vez, se ha efectuado un análisis sensorial para la selección de la receta ganadora para el consumidor final. Adicionalmente se ha podido garantizar tanto a nivel microbiológico como nutricional, que fuera un producto estable en el tiempo y cumpliera con los parámetros de calidad establecidos. Una vez definidas las etapas productivas para su conservación, se ha seleccionado la pasteurización en frío mediante altas presiones hidrostáticas, lo que ha sido clave para obtener un producto estable y de calidad. La última fase del estudio ha sido la validación pre-comercial de los formatos y de sus propiedades funcionales a través de un ensayo de intervención poblacional, para validar las alegaciones de antioxidantes y su alto contenido en fibra, mediante el análisis de plasma sanguíneo de los consumidores a distintos tiempos desde el consumo 
para determinar la capacidad antioxidante, y de la funcionalidad "alto contenido en fibra". A partir de los resultados del proyecto, Grupo Empresarial La Caña ha comenzado su nueva línea de productos funcionales de quinta gama, una nueva iniciativa empresarial, denominada Caña Nature, S.L., en la cual produce y comercializa estos productos entre otros.

Figura 2. Guacamoles funcionales

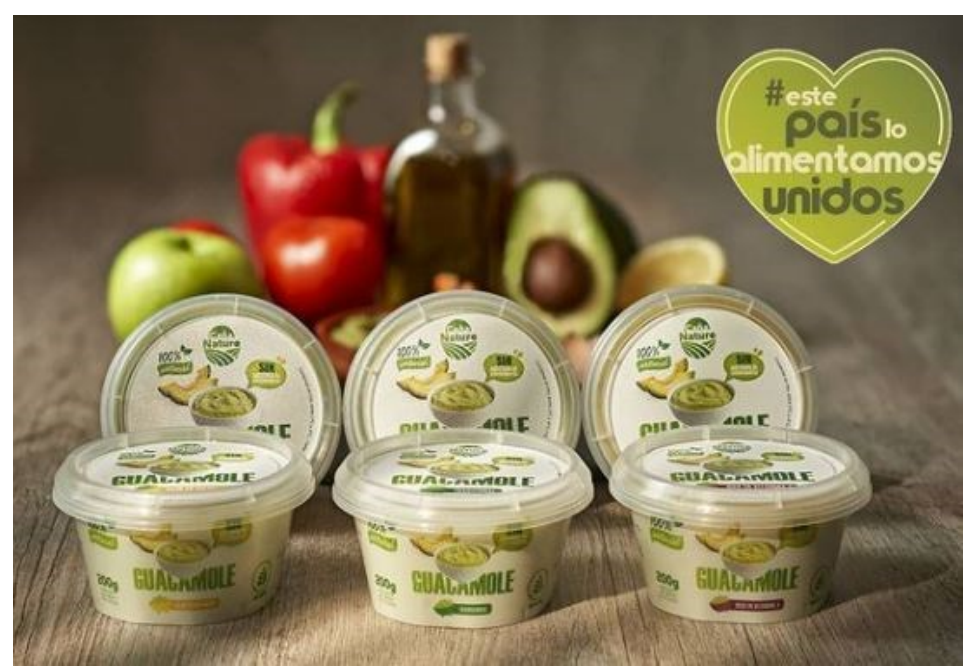

\subsection{Proyecto ACTILIFE}

Por su parte, el Proyecto ACTILIFE, aborda la interrelación entre la actividad física, la salud y la calidad de vida. Para funcionar de manera óptima y prevenir enfermedades, el cuerpo humano necesita actividad física. Debemos destacar que hay una población consciente de ello que realiza deporte para mejorar su estado físico. Unido a esto se debe llevar una dieta completa, variada y que cuente con las cantidades necesarias. Por ello se debe elaborar productos alimenticios específicos que presenten todos los macronutrientes y micronutrientes necesarios para satisfacer los requerimientos para un deportista aficionado, con el fin de contribuir al mantenimiento de su salud. Este el objetivo principal de este proyecto, que aporta además una innovación tecnológica al desarrollarse una aplicación móvil que incluya una red de conocimiento desde la medicina del deporte hasta la medicina nutricional, obtenida a través de un ensayo de intervención nutricional. Con esta información se creará un perfil 
personalizado para cada usuario según la actividad que realice, los tipos, cantidades y dosis de alimentos consumidos y momento óptimo de consumo. Por todo ello, tres empresas del sector de la alimentación, se han involucrado en el marco de este proyecto cubriendo el aporte de ingredientes alimentarios con el fin de satisfacer las necesidades y expectativas de una sociedad activa que demanda alimentos saludables junto con una herramienta de gestión digital que contribuya a lograr una vida saludable y mejorar la dieta. En la actualidad, este proyecto se encuentra en fase de producción de dos matrices alimentarias con base líquida para su consumo antes y después del deporte. Se han estudiado las necesidades nutricionales del deportista aficionado para poder definir el producto alimenticio a desarrollar y se han seleccionado los productos alimenticios a través de la caracterización de las distintas materias primas desde el punto de vista nutricional.

\subsection{Proyecto FOOD4STROKE}

Siguiendo con la estrategia de elaborar un producto alimenticio enfocado a mejorar la vida saludable de las personas, el proyecto FOOD4STROKE está enfocado al target de mercado senior, a los problemas de salud asociados al envejecimiento poblacional y a otros problemas de salud pública centrados en el aumento de la prevalencia de determinadas enfermedades crónicas, como son las enfermedades vasculares que pueden producir enfermedades neurodegenerativas. La tendencia de una sociedad consciente de este problema, ha derivado en un mayor consumo responsable de alimentos, sabiendo que una dieta equilibrada no sirve únicamente como nutrición y soporte, sino que además estos alimentos desempeñan un papel clave en la calidad de vida de las personas. Por ello nace este proyecto, de la necesidad de investigar y desarrollar nuevos alimentos funcionales, con un valor añadido, partiendo de la investigación de materias primas con las que se trabaja habitualmente en Grupo La Caña. Estas materias primas tienen un efecto neuroprotector beneficioso para la salud, con potencial para la prevención de enfermedades neurodegenerativas. 
La mayor innovación del proyecto es, una vez conocidos esos componentes funcionales, explorar las combinaciones de las materias primas seleccionadas buscando efectos sinérgicos sobre el efecto neuroprotector no explorado con anterioridad. Con el fin de reforzar esta estrategia, al tiempo que se promueve de una dieta adecuada, la industria agroalimentaria se encuentra ante el reto de desarrollar nuevos alimentos con efecto preventivo frente a estas enfermedades, sobre todo considerando que, en las últimas décadas, se ha producido un cambio en las tendencias alimentarias de los países desarrollados, y el concepto de alimentación equilibrada ha pasado a significar mantener una dieta adecuada basada en el consumo de alimentos que promuevan la salud y mejoren el bienestar de la sociedad.

\subsection{Proyecto AGUACAVALUE}

Para cerrar el círculo y como ejemplo de economía circular, se expone el proyecto AGUACAVALUE. La sociedad está cada vez más preocupada por el impacto de la actividad industrial en el medio ambiente, unido a una exigente legislación ambiental que impulsa acciones hacia una actividad más sostenible a través de prácticas y procesos respetuosos con el medio ambiente. En este caso nos centramos en los subproductos o coproductos generados en la industria agroalimentaria, definidos como productos que comercialmente no cumplen el estándar de calidad o que tienen algún tipo de defecto. En España, en general, hay mucho producto con un alto contenido de componentes bioactivos con efecto beneficioso, cuya revalorización supone una reducción del coste total del tratamiento de residuos, aumentando la sostenibilidad de los procesos productivos. Este proyecto tiene su foco puesto en el producto del aguacate, especialmente debido a que, para satisfacer su demanda, se ha visto incrementada la superficie cultivada a nivel nacional en los últimos años. Actualmente, el estilo de vida de la sociedad actual, la preocupación de salud y la falta de tiempo para cocinar, entre otros condicionantes, ha propiciado un incremento de la comercialización y del consumo de alimentos preparados. El consumo de aguacate se realiza fundamentalmente en fresco y preparado en guacamoles y salsas. En fresco se genera un 3\% del subproducto, y como 
preparado se genera un 30\%. En España se desechan aproximadamente dos mil toneladas de huevo y piel de aguacate, lo que provoca un problema para las industrias, por un lado, debido al coste económico que supone su gestión y por otro, al impacto ecológico que supone su desecho. Por ello se deben reutilizar, reducir y reciclar estas materias primas ya que son recursos bioactivos con un valor comercial y perfil saludable significativo, lo que las convierte en fuentes susceptibles para construir y revalorizar un nuevo producto procesado. La piel del aguacate está compuesta por componentes con poder antioxidante, antimicrobiano, bioadsorbente e incluso insecticida. La semilla por su parte, es incluso más rica, ya que, aparte de los poderes anteriormente comentados (a excepción de insecticida), tiene poder antihipertensivo, antidiabético, anticancerígeno, fuente de fibra dietética, efecto hipocolesterolémico, dermoprotector, colorante, espesante y biocombustible (Brown et al., 2008).

En definitiva, la revalorización de estos subproductos conduce a un aumento significativo de la sostenibilidad medioambiental de la explotación del fruto. La transformación y aprovechamiento de estos subproductos, reduce el impacto negativo que su eliminación puede generar. Los tratamientos podrían ser más o menos complejos (fertilizantes, compostaje, combustión, digestión anaerobia, etc....) siendo importante su conversión en un producto revalorizado y con mayor valor agregado. Todos estos factores pueden tener una repercusión directa sobre el empleo, al requerir de empresas biotecnológicas para su posterior aplicación en sectores como el nutracéutico, alimentario, cosmética, farmacéutico o animal. En la industria agroalimentaria, las materias primas frescas pueden tener dos vías de utilización: en fresco o procesado. Dada la elevada producción de subproductos que no son comerciales o ciertas partes del fruto que, tras ser procesados, no tengan interés para esa matriz alimentaria, va a existir un potencial impacto ambiental que debe ponerse en el foco de las industrias agroalimentarias para su correcta gestión. El gran volumen de estos subproductos con un porcentaje elevado de agua resulta desafiante, debido principalmente a la rápida degradación de dicho subproducto si éste no es secado o tratado de forma inmediata o 
adecuadamente. La existencia de estos subproductos o co-productos, tiene una gran repercusión desde el punto de vista económico, social y nutricional, especialmente ante un manejo ineficaz o inapropiado que puede implicar que se convierta en una fuente de contaminación de los recursos naturales, suelo, agua y aire. Con la transformación de estos subproductos se puede reducir notablemente este impacto, revalorizando su uso, suponiendo una fuente de oportunidades a través de la utilización y uso racional de estos componentes para su conversión en productos útiles. Para obtener un producto con valor añadido, se deben tener en cuenta varias etapas previas desde su tratamiento hasta la formulación de los productos finales, obteniendo y haciendo un seguimiento de los compuestos bioactivos a lo largo de todo el proceso.

Concienciados con la cadena agroalimentaria desde el agricultor hasta el consumidor, Grupo La Caña asume el desafío de la demanda de una producción más sostenible y amable con el medioambiente y para ello adopta tecnologías innovadoras teniendo en cuenta criterios sostenibles y de calidad y seguridad alimentaria de sus productos.

\section{REFERENCIAS}

JUNTA DE ANDALUCÍA (2018). Estrategia Andaluza de Bioeconomía Circular. Recuperado de: https://www.juntadeandalucia.es/export/drupaljda/Estrategia Andaluz a_Bioeconomia_Circular_EABC_18.09.2018.pdf

KENNETT, D.J., \& WINTERHALDER, B. (Eds.). (2006). Behavioral ecology and the transition to agriculture. Berkeley: University of California Press. 